\title{
COX-2 activation is associated with Akt phosphorylation and poor survival in ER-negative, HER2-positive breast cancer
}

\author{
Sharon A Glynn ${ }^{1,2}$, Robyn L Prueitt ${ }^{1}$, Lisa A Ridnour ${ }^{3}$, Brenda J Boersma', Tiffany M Dorsey ${ }^{1}$, David A Wink ${ }^{3}$,
} Julie E Goodman ${ }^{4}$, Harris G Yfantis ${ }^{5}$, Dong H Lee ${ }^{5}$, Stefan Ambs ${ }^{1 *}$

\begin{abstract}
Background: Inducible cyclooxgenase-2 (COX-2) is commonly overexpressed in breast tumors and is a target for cancer therapy. Here, we studied the association of COX-2 with breast cancer survival and how this association is influenced by tumor estrogen and HER2 receptor status and Akt pathway activation.

Methods: Tumor COX-2, HER2 and estrogen receptor $\alpha$ (ER) expression and phosphorylation of Akt, BAD, and caspase-9 were analyzed immunohistochemically in 248 cases of breast cancer. Spearman's correlation and multivariable logistic regression analyses were used to examine the relationship between COX-2 and tumor characteristics. Kaplan-Meier survival and multivariable Cox proportional hazards regression analyses were used to examine the relationship between COX-2 and disease-specific survival.

Results: COX-2 was significantly associated with breast cancer outcome in ER-negative [Hazard ratio (HR) $=2.72$; 95\% confidence interval (CI), 1.36-5.41; comparing high versus low COX-2] and HER2 overexpressing breast cancer $(\mathrm{HR}=2.84 ; 95 \% \mathrm{Cl}, 1.07-7.52)$. However, the hazard of poor survival associated with increased COX-2 was highest among patients who were both ER-negative and HER2-positive (HR $=5.95 ; 95 \% \mathrm{Cl}, 1.01-34.9)$. Notably, COX-2 expression in the ER-negative and HER2-positive tumors correlated significantly with increased phosphorylation of Akt and of the two Akt targets, BAD at Ser136 and caspase-9 at Ser196.

Conclusions: Up-regulation of COX-2 in ER-negative and HER2-positive breast tumors is associated with Akt pathway activation and is a marker of poor outcome. The findings suggest that COX-2-specific inhibitors and inhibitors of the Akt pathway may act synergistically as anticancer drugs in the ER-negative and HER2-positive breast cancer subtype.
\end{abstract}

\section{Background}

Cyclooxygenase-2 (COX-2) catalyzes the conversion of arachidonic acid to prostaglandin $\mathrm{E}_{2}\left(\mathrm{PGE}_{2}\right)$ and enhances the metastatic phenotype of both breast cancer cells in vitro and breast tumors [1]. Increased COX-2 expression occurs early in breast cancer and can be detected in ductal carcinoma in situ [2], invasive breast carcinoma [3] and in metastatic lesions [4]. Recently, COX-2 expression has been associated with decreased disease-free survival in breast cancer [5], and breast

\footnotetext{
*Correspondence: ambss@mail.nih.gov

'Laboratory of Human Carcinogenesis, Center for Cancer Research (CCR),

National Cancer Institute (NCI), NIH, Bethesda, Maryland (MD), USA

Full list of author information is available at the end of the article
}

cancer specific survival [6-8], suggesting that the inhibition of this enzyme has anticancer effects.

We have previously observed a significant association between COX-2 expression and Akt phosphorylation in breast tumors [9]. We also demonstrated the ability of $\mathrm{PGE}_{2}$ to induce phosphorylation of Akt in the ERnegative MDA-MB-231 breast cancer cells, and to a lesser degree in ER-positive MCF-7 breast cancer cells. The results indicated that COX-2 is a key modulator of Akt activation in breast cancer which is consistent with other published findings [10]. Additionally, it has been shown by others that administration of the COX2 inhibitor, celecoxib, in murine mammary tumor models results in inhibition of Akt phosphorylation and enhanced induction of apoptosis [11].

\section{C) Biomed Central}


In the current study, we hypothesized that COX-2 expression would be associated with poor breast cancer survival, and that the COX-2 effect on survival would be modified by the tumor ER and human epidermal growth factor receptor 2 (HER2) status and/or Akt pathway activation. A recent celecoxib anti-aromatase adjuvant trial did not find a clinical benefit for celecoxib, a COX2-specific inhibitor, among ER-positive patients with advanced disease [12], while two other similar trials showed promising, albeit not significant effects of this drug when administered in combination with exemestane $[13,14]$. We investigated the association of COX-2 expression with disease outcome among ER-positive and ER-negative breast cancer patients. The results from our study suggest that COX-2-specific inhibitors could be more efficacious in ER-negative tumors than ER-positive tumors and may perhaps synergistically interact with Akt inhibitors in breast cancer survival.

\section{Methods}

\section{Tissue collection}

Paraffin-embedded $(n=248)$ tumor specimens were obtained from breast cancer patients that resided in the greater Baltimore area, as described [9]. Patients were recruited at the University of Maryland Medical Center (UMD), the Baltimore Veterans Affairs Medical Center, Union Memorial Hospital, Mercy Medical Center, and the Sinai Hospital in Baltimore between 1993 and 2003. All patients were identified through surgery lists and enrolled into the study prior to surgery. They signed a consent form and completed an intervieweradministered questionnaire. Clinical and pathological information was obtained from medical records and pathology reports. Disease staging was performed according to the tumor-node-metastasis (TNM) system of the American Joint Committee on Cancer/the Union Internationale Contre le Cancer (AJCC/UICC). The Nottingham system was used to determine the tumor grade. The collection of tumor specimens, survey data, and clinical and pathological information was reviewed and approved by the University of Maryland Institutional Review Board for the participating institutions (UMD protocol \#0298229). IRB approval of this protocol was then obtained at all institutions (Veterans Affairs Medical Center, Union Memorial Hospital, Mercy Medical Center, and Sinai Hospital). The research was also reviewed and approved by the NIH Office of Human Subjects Research (OHSR \#2248).

\section{Immunohistochemistry}

IHC was performed as described previously [9]. Staining specificity was evaluated and shown with negative and positive control slides and, if available, with blocking peptides that were purchased from the manufacturer.
Phospho-specific blocking peptides were available for phosphorylated Akt and phosphorylated caspase-9. To block phospho-Akt staining, blocking peptides from Cell Signaling for Akt Ser473 (\#1140) and Akt Thr308 (\#1145) were used. The specificity of these blocking peptides for phospho-Akt has been shown by the manufacturer. In brief, protein expression was evaluated using the following primary antibodies: 1:50 diluted monoclonal antibody (clone 33; no. 610204 (formerly C22420); BD Biosciences/Transduction Laboratories, San Diego, CA) for COX-2; 1:100 diluted rabbit polyclonal antibody (DakoCytomation) for HER2 (c-erbB-2); and ready-touse monoclonal (Clone 6F11) antibody (Ventana Medical Systems, Tucson, AZ) for the estrogen receptor (ER); 1:25 diluted rabbit polyclonal antibody (no. 9277; Cell Signaling Technology, Beverly, MA) for phosphorylated Akt $\left(\operatorname{Ser}^{473}\right) ; 1: 80$ diluted monoclonal antibody 244F9 (no. 4056; Cell Signaling Technology) for phosphorylated Akt $\left(\mathrm{Thr}^{308}\right) ; 1: 100$ diluted rabbit polyclonal antibody (no. 9295; Cell Signaling Technology) for phosphorylated Bad (Ser $\left.{ }^{136}\right)$; 1:250 diluted rabbit polyclonal antibody (no. SC-11755; Santa Cruz Biotechnology, Santa Cruz, CA) for phosphorylated caspase-9 $\left(\operatorname{Ser}^{196}\right)$, and the ready-to-use monoclonal antibody (Lab Vision Corp., Fremont, CA) for CD31. The IHC protocol to determine the tumor ER status followed guidelines for clinical laboratories to evaluate semiquantitatively ER expression in formalin-fixed, paraffinembedded tissue on a Ventana automated slide stainer for clinical assessment of a patient's ER status ("CONFIRM Estrogen Receptor" assay by Ventana). The IHC staining protocol for HER2 followed the DAKO HercepTest $^{\mathrm{TM}}$ protocol. A combined score of intensity and distribution was used to categorize the immunohistochemical staining for protein expression with the exception of the ER IHC. Intensity received a score of 0 to 3 if the staining was negative, weak, moderate, or strong. The distribution received a score of 0 to 4 if the staining distribution was $<10 \%$ positive cells, $10 \%-30 \%$, $>30 \%$ $50 \%,>50 \%-80 \%$, and $>80 \%$. A sum score was then divided into four groups as follows: $(1)$ negative $=0-1$, (2) weak $=2-3,(3)$ moderate $=4-5$, and (4) strong = 6-7. The ER status was scored negative/positive. The ER status was determined at the Department of Pathology, University of Maryland, according to the reference range set by the $\mathrm{ChromaVision}^{\oplus} \mathrm{ACIS}^{\oplus}$ assisted quantitative image analysis software (Clarient Diagnostic Services, Irvine, CA), consistent with clinical guidelines. The HER2 status was determined using either the sum score system, as described above, or the score system according to the HercepTest ${ }^{\mathrm{Tm}}$ protocol. However, additional FISH results to detect HER2 amplification were not available for this patient cohort. The quantification of the tumor MVD was performed on CD31-positive 
microvessels according to the method of Weidner et al [15]. Microvessels were counted per 200× field in the most vascular region of the tumor.

\section{TP53 mutational analysis}

Tumors were screened for $\mathrm{p} 53$ mutations as previously described [16].

\section{Statistical analysis}

Data analysis was performed using Stata/SE 10.1 (Stata Corp, College Station, TX) statistical software package. All statistical tests were two-sided, and an association was considered statistically significant with $P$ values $<.05$. Spearman's rank correlation and logistic regression models were used for correlation analysis and to calculate odds ratios (ORs), respectively. Multivariable regression models were applied to calculate adjusted ORs. Survival was determined for the period from the date of hospital admission to the date of the last completed search for death entries in the Social Security Index (date of search: December $\left.31^{\text {st }}, 2006\right)$ for the 248 patients. The mean and median follow-up times for breast cancer survival were 71 months and 68 months, respectively (range: 12 to 166 months). A total of 89 (36\%) of these 248 patients died during this period. We obtained information (National Death Index, death certificates) on the causes of death for the deceased patients and censored all patients whose causes of death, such as accidents, were not related to breast cancer ( $\mathrm{n}=$ 11). The Kaplan-Meier survival method and the log-rank test of equality of survival function were used for univariate survival analysis. Cox regression was used for multivariable survival analysis to calculate adjusted hazard ratios. For the survival and logistic regression analyses, COX-2 expression was dichotomized into high and low. COX-2 IHC scores of moderate to strong were categorized as high and scores of negative to weak were categorized as low. The following covariates were included into the analyses: age at diagnosis (as a continuous variable), race/ethnicity (African-American versus European-American), TNM stage (categorized as $\leq$ stage II versus > stage II), tumor grade (categorized as $\leq$ grade 2 versus $>$ grade 2 ), chemotherapy (yes/no), and p53 mutation (categorized as negative versus positive). In the univariate analysis, age at diagnosis, TNM stage, tumor grade, and p53 mutational analysis were significantly associated with disease outcome. Proportional hazards assumptions were verified by log-log plots and with the nonzero slope test of the scaled Schoenfeld residuals.

\section{Results}

\section{Study population characteristics}

We have previously reported a functional relationship between tumor COX-2 expression and Akt pathway activation in breast cancer [9]. To explore this finding further, we examined the relationship between tumor COX-2 expression and disease outcome in the same patient population from the greater Baltimore area. Patient characteristics and tumor marker expression are described in Table 1. Representative immunostains for

Table 1 Demographic and clinicopathological features of cases

\begin{tabular}{|c|c|c|c|}
\hline & & $\mathbf{N}$ & $\%$ \\
\hline \multirow[t]{2}{*}{ ER Status } & Negative & 102 & $41 \%$ \\
\hline & Positive & 145 & $59 \%$ \\
\hline \multirow[t]{4}{*}{ HER2 Status } & Negative & 106 & $43 \%$ \\
\hline & Weak & 48 & $19 \%$ \\
\hline & Moderate & 51 & $21 \%$ \\
\hline & High & 42 & $17 \%$ \\
\hline \multirow[t]{2}{*}{ TNM Stage } & $<=\|$ & 184 & $71 \%$ \\
\hline & $>=\|\|$ & 44 & $29 \%$ \\
\hline \multirow[t]{2}{*}{ Grade } & 1 or 2 & 108 & $50 \%$ \\
\hline & 3 & 107 & $50 \%$ \\
\hline \multirow[t]{4}{*}{$\operatorname{cox}-2$} & Negative & 75 & $30 \%$ \\
\hline & Weak & 83 & $34 \%$ \\
\hline & Moderate & 65 & $26 \%$ \\
\hline & High & 25 & $10 \%$ \\
\hline \multirow[t]{4}{*}{ pAkt Ser473 } & Negative & 17 & $7 \%$ \\
\hline & Weak & 29 & $12 \%$ \\
\hline & Moderate & 59 & $24 \%$ \\
\hline & High & 142 & $57 \%$ \\
\hline \multirow[t]{4}{*}{ pAkt Thr308 } & Negative & 14 & $6 \%$ \\
\hline & Weak & 24 & $10 \%$ \\
\hline & Moderate & 71 & $29 \%$ \\
\hline & High & 134 & $55 \%$ \\
\hline \multirow[t]{4}{*}{ pCaspase9 Ser196 } & Negative & 38 & $15 \%$ \\
\hline & Weak & 30 & $12 \%$ \\
\hline & Moderate & 73 & $30 \%$ \\
\hline & High & 106 & $43 \%$ \\
\hline \multirow[t]{4}{*}{ pBAD Ser136 } & Negative & 43 & $18 \%$ \\
\hline & Weak & 41 & $17 \%$ \\
\hline & Moderate & 76 & $31 \%$ \\
\hline & High & 85 & $34 \%$ \\
\hline \multirow[t]{3}{*}{ Survival } & Alive & 159 & $64 \%$ \\
\hline & Death from breast cancer & 78 & $32 \%$ \\
\hline & Death from other causes & 11 & $4 \%$ \\
\hline \multirow[t]{2}{*}{ Race } & $\mathrm{AA}$ & 143 & $58 \%$ \\
\hline & EA & 105 & $42 \%$ \\
\hline \multirow[t]{2}{*}{ p53 mutation } & Negative & 200 & $81 \%$ \\
\hline & Positive & 48 & $19 \%$ \\
\hline \multirow[t]{3}{*}{ Chemotherapy } & No & 99 & $43 \%$ \\
\hline & Yes & 132 & $57 \%$ \\
\hline & & \multicolumn{2}{|c|}{ mean $\pm S D$} \\
\hline Age at Diagnosis & $(n=248)$ & \multicolumn{2}{|c|}{$55.0 \pm 13.9$} \\
\hline Body mass index ${ }^{1}$ & $(n=236)$ & \multicolumn{2}{|c|}{$29.0 \pm 8.1$} \\
\hline CD31 & $(n=208)$ & \multicolumn{2}{|c|}{$49.1 \pm 43.9$} \\
\hline
\end{tabular}

${ }^{1}$ At time of hospital admission. 
COX-2, phosphorylated Akt, BAD, and caspase- 9 are shown in Figures $1 \& 2$ and for HER2 in Figure 3. TNM stage, tumor grade, tumor ER and HER2 status, patient's race/ethnicity, or age at diagnosis were not associated with COX-2 expression in the tumor. However, COX-2 expression was found to be inversely correlated with body mass index at hospital admission. After stratification by tumor ER status, this association was restricted to patients with ER-negative breast cancer (Spearman's correlation coefficient: $-0.24 ; p=0.024$ ).

\section{COX-2 expression predicts poor disease-specific survival} in both ER-negative and HER2 expressing breast cancers High COX-2 expression was significantly associated with inferior breast cancer-specific survival in ER-negative patients $(p=0.001)$ (Figure 4$)$. We did not find an association between COX-2 and survival in patients with ER-positive tumors in this population $(p=0.483)$. COX2 was also associated with a significantly decreased survival in patients with high HER2 (HER2-positive) tumors $(p=0.022)$ but not with survival in patients with low HER2 (HER2-negative) breast tumors $(p=0.601)$ (Figure 4). A similar trend was observed when the tumor HER2 status was determined immunohistochemically according to HercepTest ${ }^{\mathrm{TM}}$ guidelines. Because FISH results to detect HER2 amplification were not available for this patient cohort, only a HercepTest ${ }^{\mathrm{TM}}$ score of 3 was considered positive for tumor HER2 expression in this analysis. There was no significant association of COX-2 with survival in patients being scored negative for HER2 (HercepTest ${ }^{\mathrm{TM}}$ score of 0 or 1), but there was a significant poorer outcome among patients with high COX-2 and a positive HercepTest ${ }^{\mathrm{TM}}$ score [Log-rank test: $p=0.017 ; \mathrm{HR}=3.2,95 \%$ CI (1.24-9.21) comparing high COX-2 $(n=24)$ versus low COX-2 $(n=29)$ in patients with a HercepTest ${ }^{\text {TM }}$ score of 3]. Multivariable Cox regression survival analysis at 5-year and 10-year followup confirmed these findings (Table 2), indicating that COX-2 was an independent predictor of poor outcome in both ER-negative and HER2-positive breast cancers at 5-year [adj. $\mathrm{HR}_{\mathrm{ER}-}=2.79$, 95\% CI (1.35-5.78); adj. $\mathrm{HR}_{\text {HER2+ }}=3.39,95 \% \mathrm{CI}$ (1.24-9.21)] and 10-year [adj. $\mathrm{HR}_{\mathrm{ER}-}=2.72,95 \% \mathrm{CI} 1.36-5.41$; adj. $\mathrm{HR}_{\mathrm{HER} 2+}=2.84$, 95\% CI 1.07-7.52] follow-up.

While COX-2 remained a predictor of poor outcome in ER-negative patients who were also HER2-negative [adj. $\mathrm{HR}=2.34,95 \%$ CI 1.01-5.41], the hazard of poor survival associated with increased COX-2 was highest in ER-negative patients who were HER2-positive [adj. HR $=5.95,95 \%$ CI 1.01-34.9], as shown by univariate (Figure 5) and multivariable analyses (Table 2). ER-/HER2+ tumors belong to a distinct subtype of breast cancer based on its gene expression profile [17]. In ER-positive tumors, no statistically significant association between
COX-2 and patient survival was observed, regardless of HER2 status, in our patient population.

\section{Relationship between COX-2 and the phosphorylation status of Akt, caspase-9 and BAD is dependent on tumor ER and HER2 status}

In a previous study of inflammation and breast cancer, we had found a positive correlation between COX-2 expression and increased phosphorylation of Akt, caspase-9 and BAD in breast tumors [9]. Both caspase-9 and BAD are downstream targets of Akt and their phosphorylation inhibits their pro-apoptotic function. Because of our observation that COX-2 is an independent predictor of poor survival in ER-negative and HER2-positive breast tumors, we applied correlation and logistic regression analyses to examine the influence of both the tumor ER and HER2 status on the association between COX-2 and Akt pathway activation in 248 breast tumors.

The correlation analysis revealed that the strongest correlation between increased COX-2 expression and Akt pathway activation is present in tumors that are ER-negative and HER2-positive (pAkt Ser473 $p=0.003$; pAkt Thr308 $p=0.003$ ) (Table 3 ). In this tumor subtype, COX-2 was also associated with a significantly increased vessel density, as judged by the number of CD31-positive microvessels $(p=0.021)$. The same associations were either weaker or absent (CD31) in all other strata supporting the hypothesis that the poor outcome signature of COX-2 in ER-negative and HER2positive breast tumors involves Akt pathway activation and increased tumor angiogenesis. Further analysis of the tumor immunohistochemistry by multivariable logistic regression corroborated the results of the Spearman's correlation analysis showing the strongest association between COX-2 expression and Akt pathway activation in ER-negative and HER2-positive breast tumors independent of age at diagnosis, disease stage, race/ethnicity, and neoadjuvant chemotherapy (Table 4). The direction and magnitude of the adjusted odds ratios point to a pathway in which COX-2 induces the phosphorylation of Akt and downstream targets most efficiently in HER2-positive breast tumors, and this effect is increased in an additive manner by an ER-negative tumor status. Furthermore we observed a statistical interaction on survival between COX-2 and pAkt Ser473 $(p<0.001)$, COX-2 and pBad Ser136 ( $p<0.001)$, COX-2 and pCasp9 Ser196 $(p<0.001)$, further indicating a significant role of Akt signaling in poor survival of COX-2 expressing tumors.

\section{Discussion}

In our study of 248 women with incident breast cancer from the Greater Baltimore area, increased expression of 


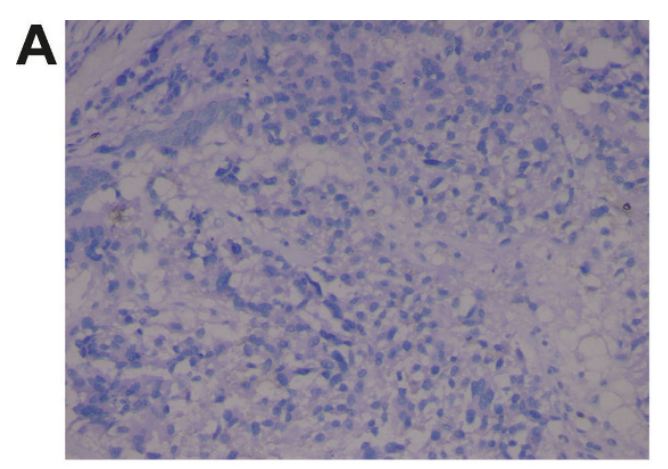

$\mathbf{E}$

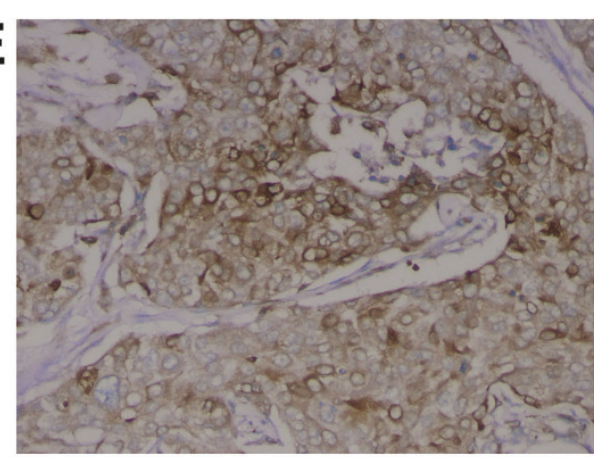

B

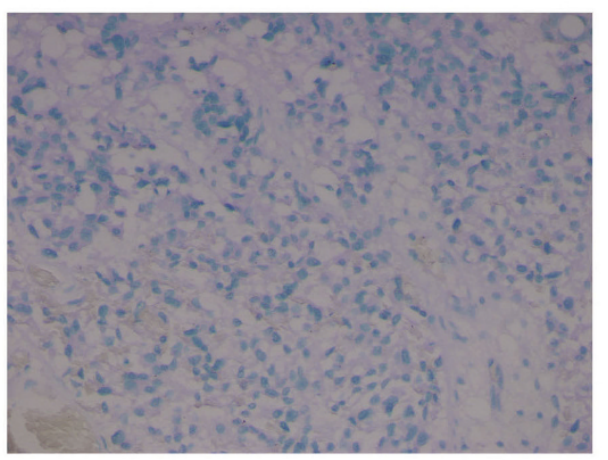

C

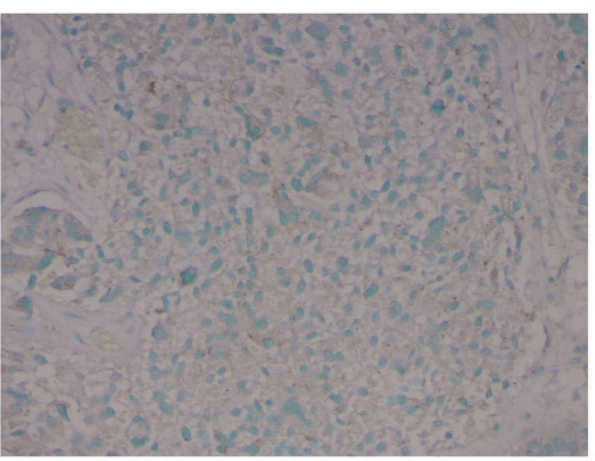

D

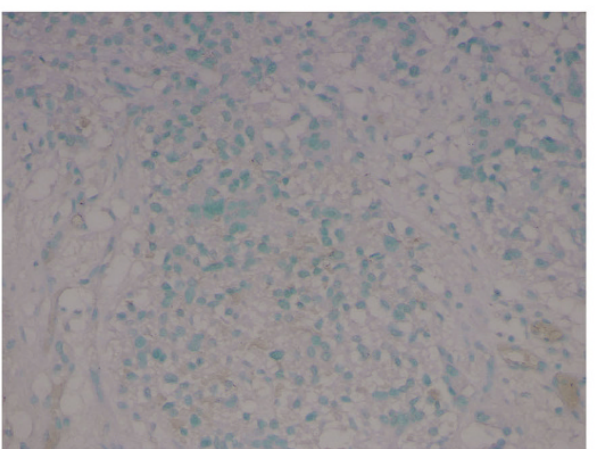

$\mathbf{F}$

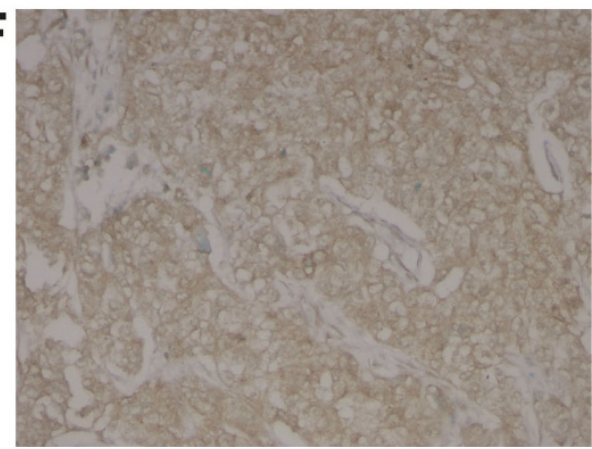

G

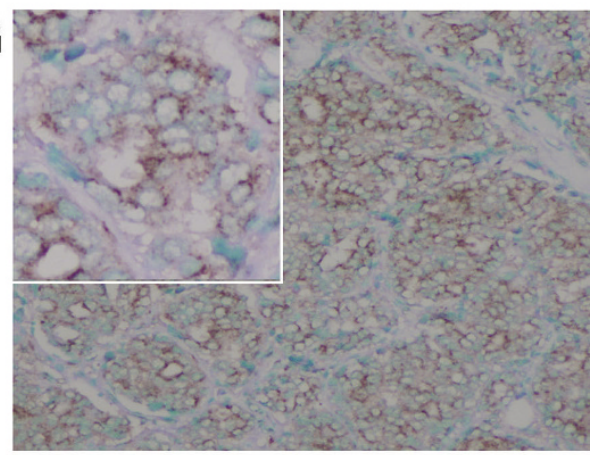

H

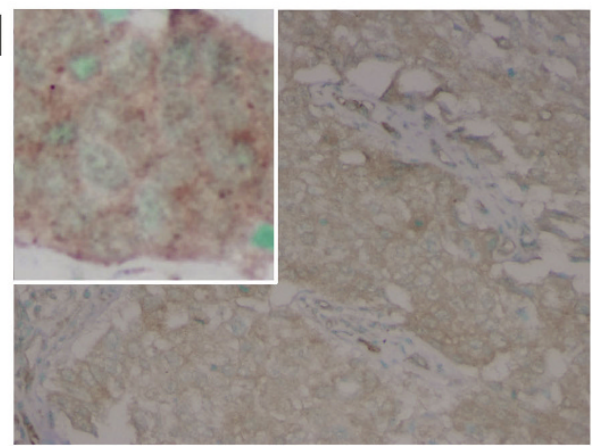

Figure 1 COX-2 expression and phosphorylation of Akt at Ser473, BAD at Ser136 and caspase-9 at Ser196 in human breast tumors. IHC analysis of two invasive carcinomas for COX-2 (A,E), Ser473-phosphorylated Akt (B,F), Ser136-phosphorylated BAD (C,G), and Ser196phosphorylated caspase-9 $(D, H)$. Tumor to the left was negative for all markers $(A, B, C, D)$. Tumor to the right shows that COX-2 is expressed in the cytoplasm of tumor cells with some cells showing marked perinuclear localization of COX-2 protein (E). Immunostain intensity is moderate to strong. Same tumors shows increased phosphoryation of Akt in the cytoplasm and along the inner cell membrane (F), and moderate to strongly increased phosphorylation of BAD $(\mathrm{G})$ and caspase-9 $(\mathrm{H})$, with a distinct immunostain suggesting a partly mitochondrial localization of the phosphorylated proteins (insets). Magnification: 200x. Counterstain: Methyl Green. 


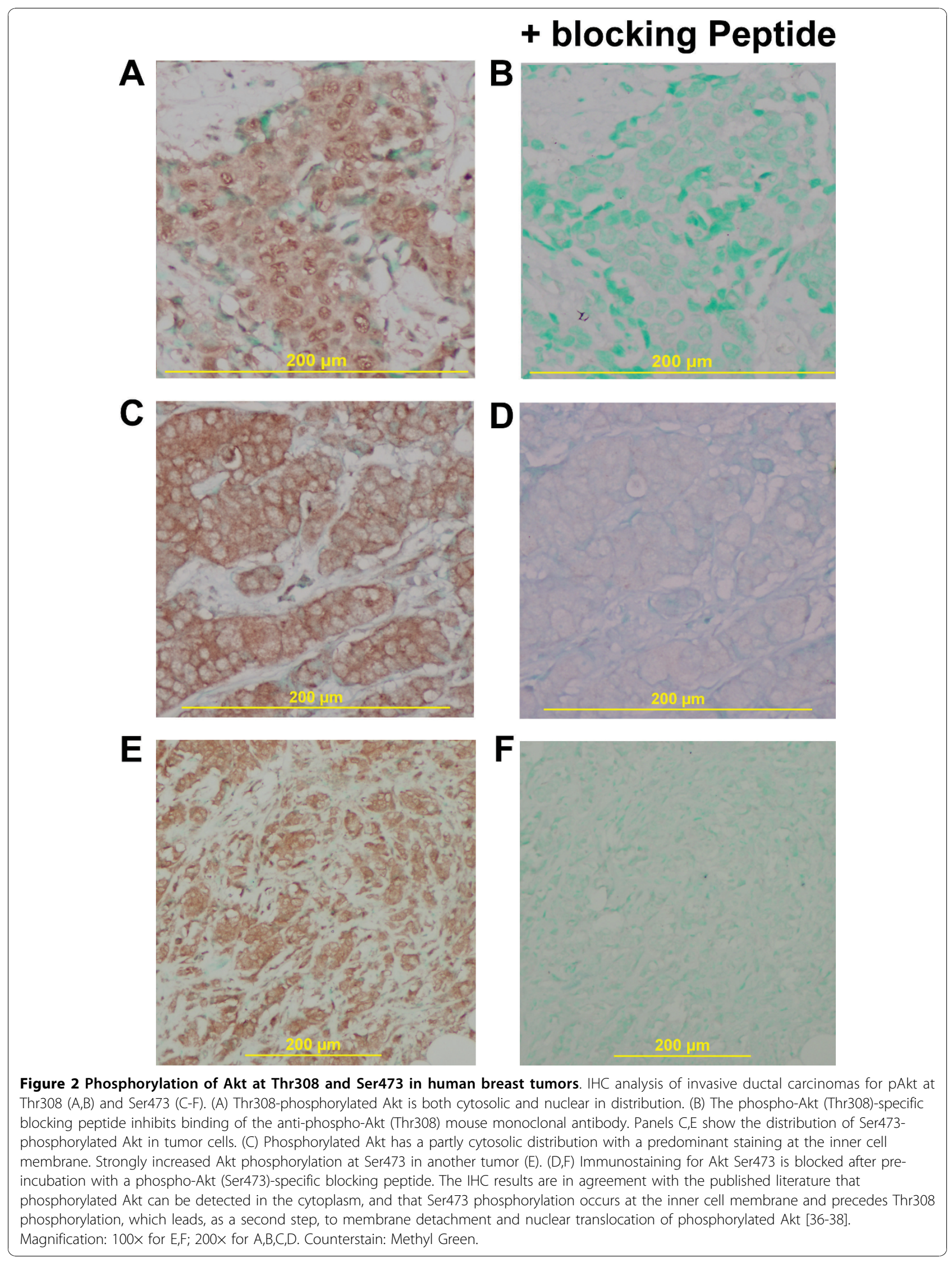




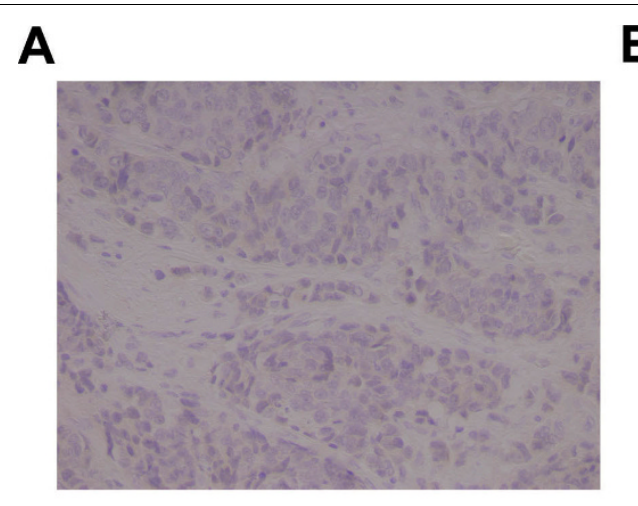

B
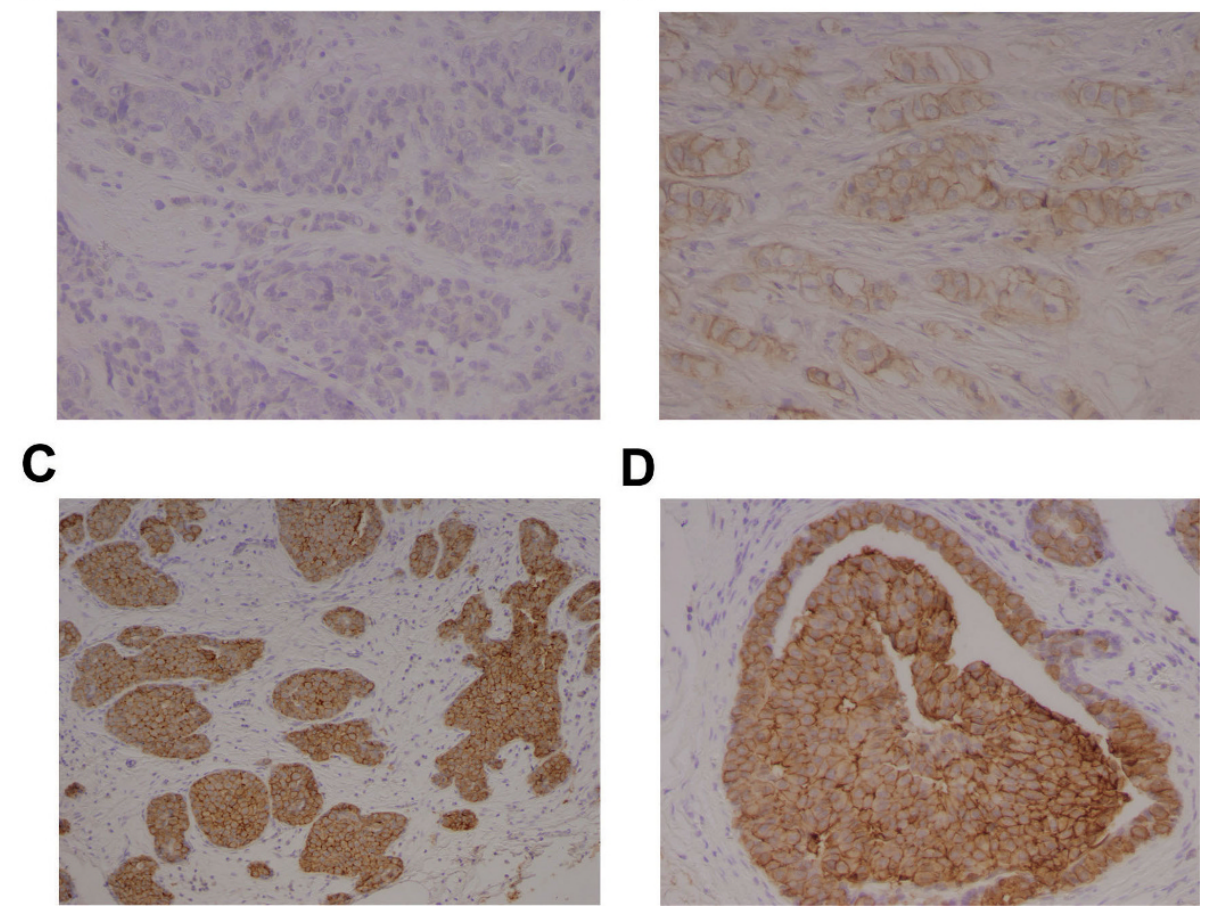

D

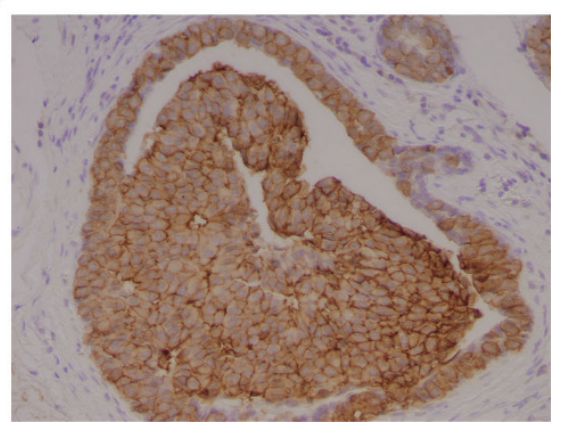

Figure 3 HER2 overexpression in breast tumors. Representative IHC staining for HER2 expression in invasive breast carcinomas (A-D). HER2 is

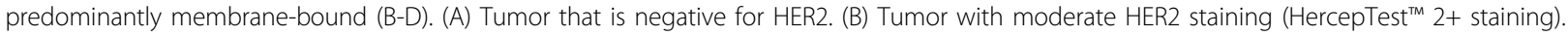
(C,D) Tumor with strong HER staining (HercepTest ${ }^{\text {TM }} 3+$ staining). Magnification: 100× for C; $200 \times$ for A,B,D. Counterstain: Hematoxylin.

COX-2 was associated with decreased breast cancerspecific survival in patients with ER-negative and HER2positive tumors, respectively. In patients with both ER-negative and HER2-positive tumors, increased tumor COX-2 was associated with the most inferior survival among all patient groups, as judged by the hazard ratio in the multivariable analysis. This was accompanied by increased Akt pathway activation, as judged by the phosphorylation status of Akt and two key downstream targets in the apoptosis pathway. These findings could have implications for COX-2 targeted therapy in breast cancer and suggest that patients with ER-negative and HER2-positive tumors would benefit from a COX-2 targeted therapy with the efficacy of this therapy being strongest in patients with both an ER-negative tumor status and an amplification of HER2 leading to high HER2 expression.

Two recent randomized clinical trials examined the efficacy of the COX-2 inhibitor celecoxib in combination with the aromatase inhibitor, exemestane, in postmenopausal women with hormone sensitive metastatic breast cancer [12,18]. Both trials failed to find a significant clinical benefit with the addition of celecoxib to the exemestane regimen, despite earlier indications in small feasibility studies that there could be increased efficacy for the combination $[13,14]$. Both trials were performed in predominantly hormone receptor-positive patients. HER2 status information was also available for the Falandry trial [18], with only $4.5 \%$ being HER2-positive. The findings in our patient population suggest that the efficacy of COX-2 inhibitors could be quite limited in ER-positive breast cancer, consistent with the trial results by Dirix et al. [12] and Falandry et al [18], suggesting that the benefit of these inhibitors as a therapeutic could be strongest in the ER-negative or HER2positive disease. Our observation of an association between COX-2 expression with poor survival in ERnegative breast cancer is in agreement with the findings of Witton et al. [19], but are different to the findings of Ristimaki et al. [5], who found that the association of COX-2 expression with distant disease-free survival was restricted to patients with ER-positive or HER2-negative breast cancer. However, there are several differences in the evaluated patients between those in the study of Ristimaki et al and our study, which may contribute to the different findings. All breast cancer patients in our study were recruited as incident cases with surgery, and additionally our study recruited both African-American and European-American patients. The Ristimaki study (recruited 1991-1992) contained solely Finnish patients, 


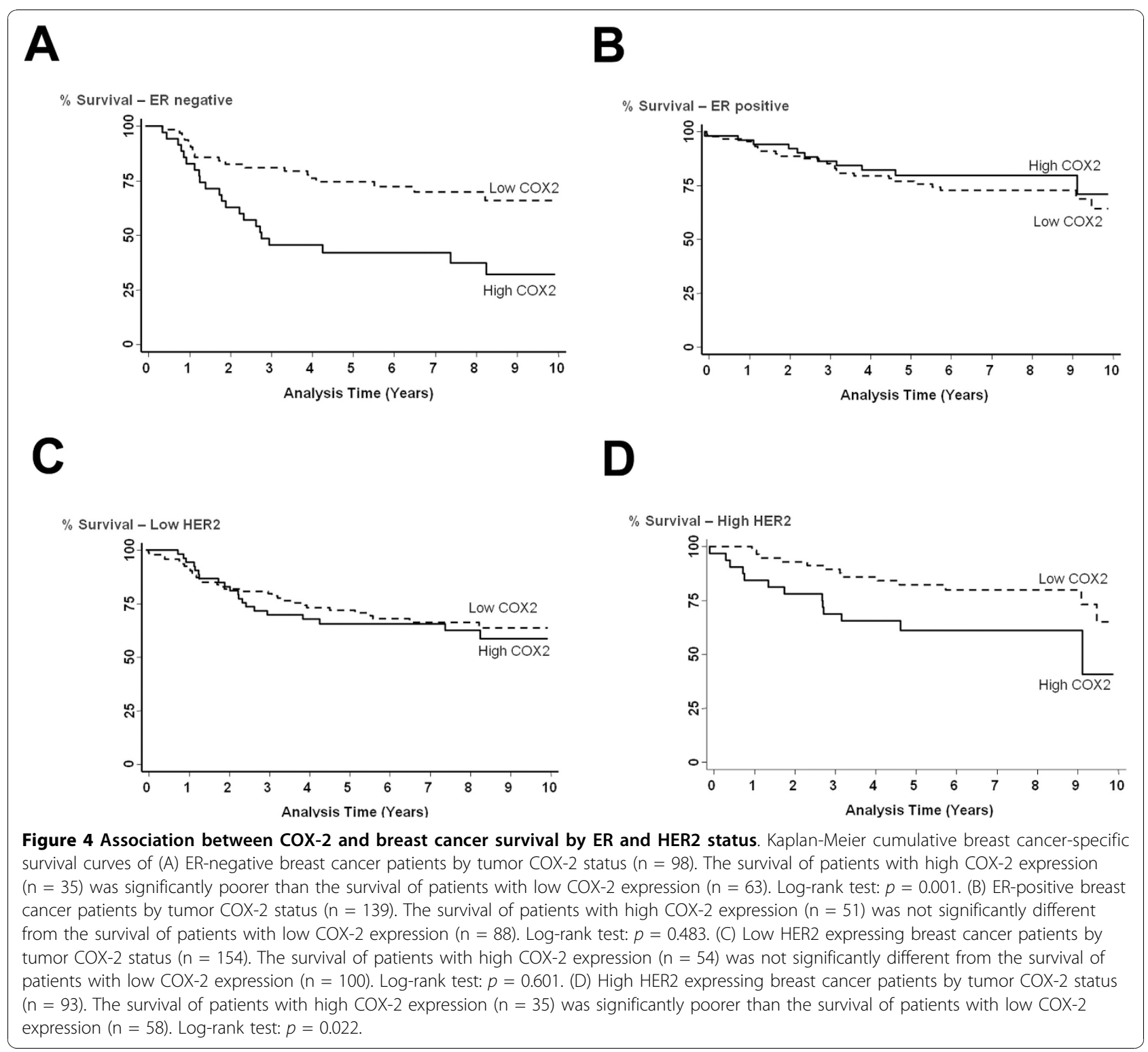

Table 2 Effects of high COX-2 expression on breast cancer survival ${ }^{1}$

\begin{tabular}{|c|c|c|c|c|c|c|c|c|}
\hline & \multicolumn{4}{|c|}{ 5-Year Multivariable Cox Regression ${ }^{3}$} & \multicolumn{4}{|c|}{ 10-Year Multivariable Cox Regression ${ }^{3}$} \\
\hline & H.R. & $95 \% \mathrm{Cl}$ & $p$-value & $\mathbf{N}$ & H.R. & $95 \% \mathrm{Cl}$ & $p$-value & $\mathbf{N}$ \\
\hline All patients & 1.82 & $1.07-3.10$ & $0.028^{*}$ & 184 & 1.60 & $0.96-2.65$ & 0.066 & 184 \\
\hline ER-negative & 2.79 & $1.35-5.78$ & $0.006^{*}$ & 81 & 2.72 & $1.36-5.41$ & $0.004^{*}$ & 81 \\
\hline ER-positive & 1.26 & $0.53-2.99$ & 0.598 & 103 & 0.96 & $0.42-2.21$ & 0.920 & 103 \\
\hline HER2-negative & 1.50 & $0.77-2.94$ & 0.237 & 121 & 1.37 & $0.73-2.56$ & 0.331 & 121 \\
\hline HER2-positive ${ }^{2}$ & 3.39 & $1.24-9.21$ & $0.017^{*}$ & 62 & 2.84 & $1.07-7.52$ & $0.036^{*}$ & 62 \\
\hline ER-/HER2- & 2.47 & $1.00-6.10$ & $0.048^{*}$ & 61 & 2.34 & $1.01-5.41$ & $0.047^{*}$ & 61 \\
\hline ER-/HER2+ & 5.95 & $1.01-34.9$ & $0.048^{*}$ & 20 & $5.95^{3}$ & $1.01-34.9$ & $0.048^{*}$ & 20 \\
\hline ER+/HER2- & 0.73 & $0.21-2.58$ & 0.626 & 60 & 0.64 & $0.18-2.20$ & 0.474 & 60 \\
\hline ER+/HER2+ & 2.60 & $0.47-14.2$ & 0.272 & 42 & 1.91 & $0.42-8.57$ & 0.397 & 42 \\
\hline
\end{tabular}

${ }^{1}$ High COX-2 expression (moderate to strong IHC) versus low COX-2 expression (absent to weak IHC).

${ }^{2}$ HER2-positive: moderate to high IHC. ${ }^{3}$ Adjusted for age at diagnosis, race, TNM stage, tumor grade, chemotherapy and tumor ER and p53 mutation status.

${ }^{3}$ No further deaths after 5 years. ${ }^{*} p<0.05$. 


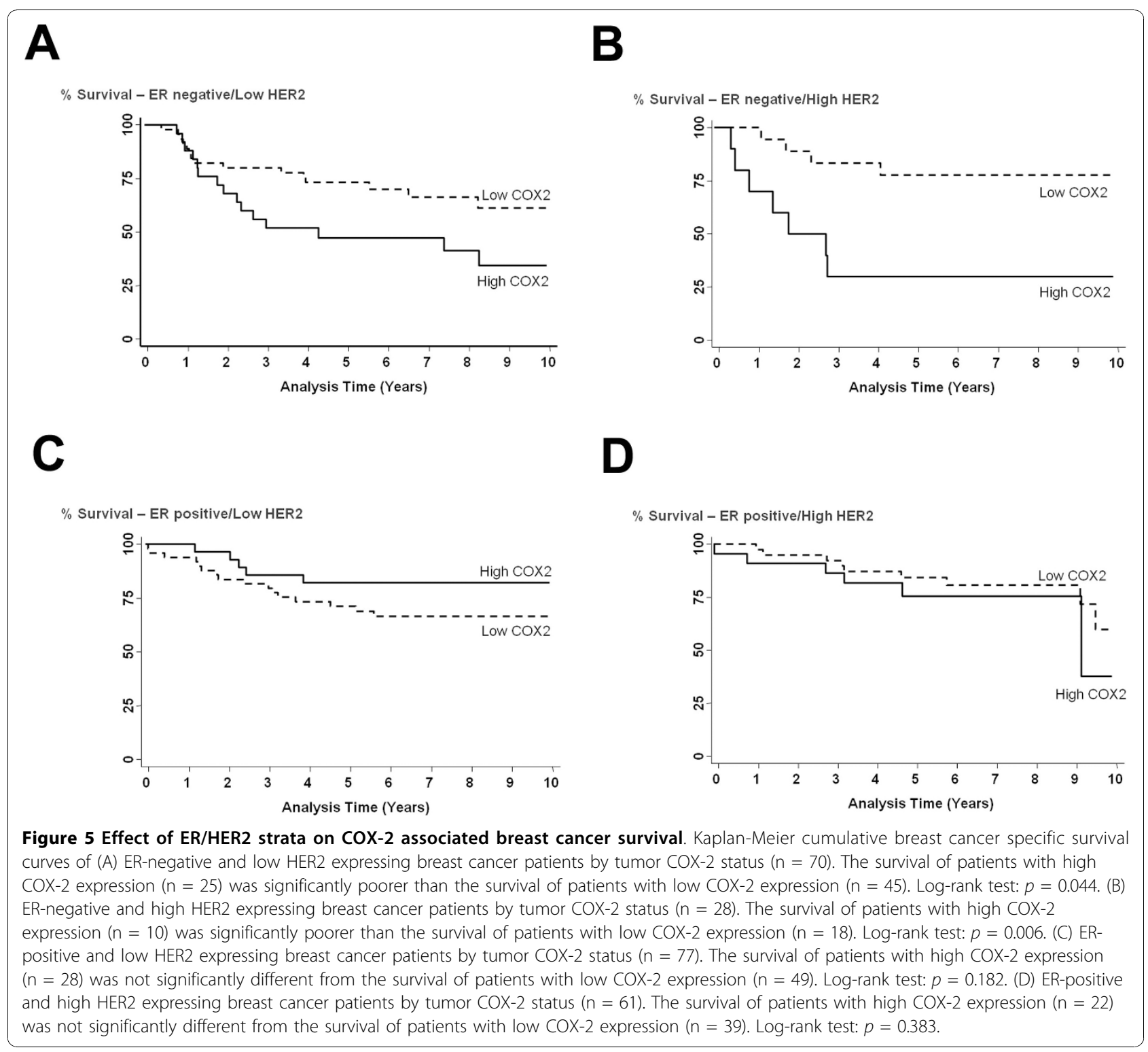

with some of them having recurrent disease. There were also differences in receipt of therapy with $47 \%$ of patients from our study versus $61 \%$ in the Ristimaki study who received postoperative radiotherapy. Of the patients with node negative disease, $75 \%$ of our patients received either adjuvant chemotherapy or endocrine therapy, versus $9 \%$ in the Ristimaki study. There were also differences in the types of therapies received. In addition, more patients in our study had high grade disease (50\%), ER-negative disease (41\%) and

Table 3 Association of COX-2 expression with tumor characteristics by tumor receptor status ${ }^{1}$

\begin{tabular}{|c|c|c|c|c|c|c|c|c|c|c|c|c|}
\hline \multirow[b]{2}{*}{ Spearman rank correlation } & \multicolumn{2}{|c|}{ All tumors } & \multicolumn{2}{|c|}{ ER-negative } & \multicolumn{2}{|c|}{ ER-positive } & \multicolumn{2}{|c|}{ Her2 low } & \multicolumn{2}{|c|}{ Her2 high } & \multicolumn{2}{|c|}{ ER-negative/HER2 high } \\
\hline & $\rho$ & $p$-value & $\rho$ & $p$ & $\rho$ & $p$ & $\rho$ & $p$ & $\rho$ & $p$ & $\rho$ & $p$ \\
\hline pAkt Ser473 & 0.23 & $<0.001$ & 0.24 & 0.011 & 0.22 & 0.006 & 0.18 & 0.024 & 0.33 & 0.001 & 0.50 & 0.003 \\
\hline pAkt Thr308 & 0.26 & $<0.001$ & 0.25 & 0.009 & 0.26 & 0.001 & 0.23 & 0.003 & 0.36 & $<0.001$ & 0.51 & 0.003 \\
\hline pCasp9 Ser196 & 0.30 & $<0.001$ & 0.31 & 0.001 & 0.30 & $<0.001$ & 0.25 & 0.001 & 0.40 & $<0.001$ & 0.67 & $<0.001$ \\
\hline pBAD Ser136 & 0.20 & 0.001 & 0.26 & 0.008 & 0.15 & 0.063 & 0.13 & 0.084 & 0.31 & 0.002 & 0.57 & $<0.001$ \\
\hline CD31 & 0.05 & 0.402 & 0.10 & 0.302 & 0.01 & 0.85 & -0.02 & 0.799 & 0.19 & 0.084 & 0.41 & 0.021 \\
\hline
\end{tabular}

\footnotetext{
${ }^{1}$ Spearman's correlation coefficient. Calculated with IHC scores 1-4 (negative, weak, moderate, strong) and continuous data (CD31).
} 


\begin{tabular}{|c|c|c|c|c|}
\hline & $\mathrm{OR}^{*}$ & $95 \% \mathrm{Cl}$ & $p$-value & $\mathrm{N}$ \\
\hline \multicolumn{5}{|c|}{ COX-2 and pAkt Ser473 } \\
\hline All patients & 1.52 & $1.05-2.09$ & 0.024 & 211 \\
\hline$E R_{N E G}$ & 1.47 & $0.84-2.57$ & 0.181 & 89 \\
\hline ERpos & 1.57 & $0.96-2.57$ & 0.072 & 121 \\
\hline HER2 ${ }_{N E G}$ & 1.33 & $0.87-2.02$ & 0.183 & 135 \\
\hline HER2pos & 2.99 & $1.09-8.15$ & 0.034 & 76 \\
\hline $\mathrm{ER}_{\mathrm{NEG}} / \mathrm{HER} 2_{\mathrm{POS}}$ & 4.87 & $0.90-26.2$ & 0.065 & 25 \\
\hline \multicolumn{5}{|c|}{ COX-2 and pAkt Thr308 } \\
\hline All patients & 1.99 & $1.31-3.02$ & 0.001 & 207 \\
\hline $\mathrm{ER}_{\mathrm{NEG}}$ & 1.85 & $0.98-3.50$ & 0.057 & 87 \\
\hline ERPOS & 2.08 & $1.18-3.63$ & 0.011 & 119 \\
\hline HER2NEG & 1.76 & $1.09-2.86$ & 0.021 & 134 \\
\hline HER2POS & 4.25 & $1.44-12.6$ & 0.009 & 72 \\
\hline $\mathrm{ER}_{\mathrm{NEG}} / \mathrm{HER} 2 \mathrm{POS}$ & 14.9 & $1.43-154$ & 0.024 & 23 \\
\hline \multicolumn{5}{|c|}{ COX-2 and pCasp9 Ser196 } \\
\hline All patients & 1.90 & $1.36-2.65$ & 0.000 & 211 \\
\hline$E R_{N E G}$ & 1.84 & $1.06-3.22$ & 0.030 & 89 \\
\hline ERpos & 2.01 & $1.31-3.08$ & 0.001 & 121 \\
\hline HER2 NEG $_{\text {N }}$ & 1.56 & $1.06-2.29$ & 0.025 & 135 \\
\hline HER2pos & 3.35 & $1.41-7.91$ & 0.006 & 76 \\
\hline $\mathrm{ER}_{\mathrm{NEG}} / \mathrm{HER} 2_{\mathrm{POS}}$ & 16.3 & $1.72-154.6$ & 0.015 & 25 \\
\hline \multicolumn{5}{|c|}{ COX-2 and pBAD Ser136 } \\
\hline All patients & 1.61 & $1.20-2.16$ & 0.001 & 209 \\
\hline $\mathrm{ER}_{\mathrm{NEG}}$ & 1.58 & $1.04-2.38$ & 0.032 & 89 \\
\hline ERPOS & 1.66 & $1.09-2.54$ & 0.019 & 119 \\
\hline HER2NEG & 1.42 & $0.99-2.02$ & 0.051 & 135 \\
\hline HER2pos & 2.21 & $1.22-3.95$ & 0.008 & 74 \\
\hline $\mathrm{ER}_{\text {NEG }} / \mathrm{HER} 2 \mathrm{POS}$ & 4.10 & $1.15-14.7$ & 0.030 & 25 \\
\hline
\end{tabular}

* OR (Odds ratio) adjusted for age at diagnosis, race, TNM stage and receipt of neoadjuvant chemotherapy.

HER2-positive disease (38\%), while in the Finnish study only $30 \%$ were diagnosed with high grade breast cancer, $31 \%$ had ER-negative disease and 18\% had HER2 amplification.

HER2 overexpression/amplification is an established marker of poor prognosis in both early [20] and late [21] stage breast cancer. HER2 overexpression is also associated with an increased risk of metastasis [22,23] and a poor response to chemotherapy in the metastatic setting $[24,25]$. Several therapies directed at the inhibition of HER2 are currently in use, including the recombinant humanized monoclonal antibodes against HER2, trastuzumbab (Herceptin ${ }^{\text {Tw}}$, Genentech), and pertuzumab (Omnitarg ${ }^{\mathrm{mix}}$, Genentech), and the small molecule tyrosine kinase inhibitor of both HER2 and EGFR Lapatinib (Tykerb $^{\text {Tw }}$, GlaxoSmithKline) [26]. Akt phosphorylation is associated with HER2 expression in breast cancer $[27,28]$, and it has been shown that HER2 transfection of MCF-7 cells leads to Akt phosphorylation mediated through the PI3K pathway [29]. These observations are consistent with the findings in our study that Akt phosphorylation in breast tumors is significantly associated with HER2 overexpression in the tumors. Additionally Akt phosphorylation has been shown to be associated with COX-2 expression in several studies [2] and COX2 specific inhibitors were found to disrupt Akt signaling in breast cancer cells [30]. We recently demonstrated that prostaglandin $\mathrm{E}_{2}\left(\mathrm{PGE}_{2}\right)$, the most active proinflammatory metabolite of COX-2, induces phosphorylation of Akt at Ser473 and GSK-3 $\beta$ at Ser9, a known downstream target of pAkt [9]. Our observation that the correlation of COX-2 with Akt pathway activation is greatest in ER-negative patients, who also have HER2 positivity, are supported by our previous finding that while $\mathrm{PGE}_{2}$ phosphorylated Akt at Ser473 and GSK-3 $\beta$ at Ser9 in two breast cancer cell lines, the response was considerably stronger in the ER-negative MDA-MB-231 than the ER-positive MCF-7 breast cancer cells. The result would suggest that ER-negative breast cancer cells may be more sensitive to Akt activation by $\mathrm{PGE}_{2}$ than ER-positive breast cancer cells. In summary, existing data suggest that breast cancers, which are ER-negative and overexpress both HER2 and COX-2, may have a more prominent Akt pathway activation, increased resistance to apoptosis, and a higher metastatic potential, which is all consistent with the findings in this study.

Of further interest is that HER2-positive breast tumorigenesis may be modulated in part by $\mathrm{COX}-2$, and vice versa. Celecoxib was found to significantly reduce mammary tumor development in HER2/neu-induced experimental mouse models in two separate studies [31,32]. Additionally, tumor multiplicity and size was significantly reduced in the HER2/neu transgenic mice, crossed with a COX-2-deficient background [1]. This suggests that COX-2 significantly contributes to HER2 associated breast tumor development. Wang et al. demonstrated the ability of nuclear HER2 to transactivate COX-2 in colon cancer cells by binding to its promoter region, thus upregulating expression of $\mathrm{COX}-2$ [33], while HER2 has also be shown to up-regulate COX-2 expression through Ras $\rightarrow$ Raf $\rightarrow$ MAPK $\rightarrow$ AP1 mechanisms in breast cancer cells [34]. Both HER2 and COX-2 expression in breast cancer cells lead to the activation of the Akt pathway. Conversely COX-2 and its product $\mathrm{PGE}_{2}$ both lead to induction of HER2 gene and protein expression in MCF-7 breast cancer cells [35]. These data indicate that a positive feedback loop exists between COX-2 and HER2 in breast cancer cells.

Our study has strengths and limitations. We conducted the analysis in 248 cases of incident breast cancer, recruiting both European-Americans and African-Americans allowing us to assess the impact of COX-2 on survival in both patient populations. We 
found that COX-2-related survival was independent of race/ethnicity, indicating that COX-2 inhibition should be equally efficacious in both patient populations. We were also able to assess the implication of the tumor p53 mutational status on the association between COX2 and breast cancer survival and observed that COX-2related survival was independent of the p53 mutation status. However, the existing sample size did not allow a more in-depth examination of the effect of race and p53 mutation status on survival in the context of increased COX-2. Furthermore, our immunohistochemical analysis of phosphorylated Akt cannot differentiate between the three Akt isoforms and FISH results to detect HER2 amplification were not available for this patient cohort. We also realize that some clinically important subgroups in our analysis were small, e.g. the number of patients with ER-/HER + tumors with high COX-2 was $n=10$, meaning that results for these subgroups should be interpreted with caution. Thus, additional studies are needed to further corroborate our findings.

\section{Conclusions}

Our study suggests that COX-2 may be a therapeutic target for the treatment of ER-negative breast cancer. A combination of COX-2, HER2, and Akt inhibitors may be particularly efficacious in patients with ER-negative/ HER2-positive breast cancer.

\section{List of Abbreviations}

COX-2: cyclooxygenase-2; ER: estrogen receptor $\alpha$; HR: hazard ratio; $\mathrm{Cl}$ : confidence interval; $\mathrm{PGE}_{2}$ : prostaglandin $\mathrm{E}_{2} ; \mathrm{TNM}$ stage: tumor-nodemetastasis stage; OR: odds ratio; HER2: human epidermal growth factor receptor 2

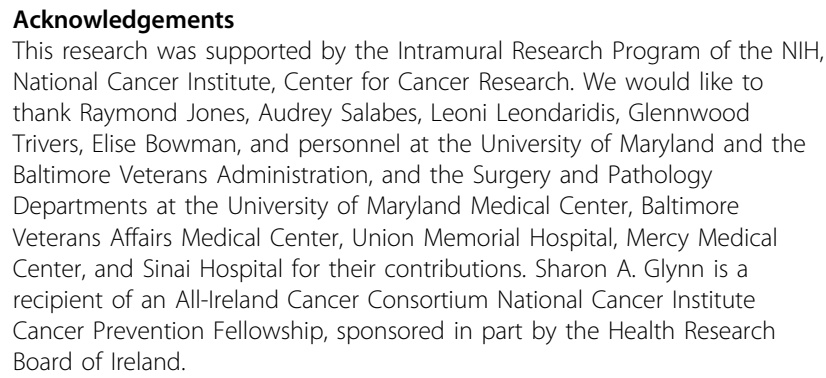

This research was supported by the Intramural Research Program of the $\mathrm{NIH}$ National Cancer Institute, Center for Cancer Research. We would like to thank Raymond Jones, Audrey Salabes, Leoni Leondaridis, Glennwood Trivers, Elise Bowman, and personnel at the University of Maryland and the Baltimore Veterans Administration, and the Surgery and Pathology Departments at the University of Maryland Medical Center, Baltimore Veterans Affairs Medical Center, Union Memorial Hospital, Mercy Medical Center, and Sinai Hospital for their contributions. Sharon A. Glynn is a recipient of an All-Ireland Cancer Consortium National Cancer Institute Cancer Prevention Fellowship, sponsored in part by the Health Research Board of Ireland.

\begin{abstract}
Author details
${ }^{1}$ Laboratory of Human Carcinogenesis, Center for Cancer Research (CCR), National Cancer Institute (NCI), NIH, Bethesda, Maryland (MD), USA. ${ }^{2}$ Cancer Prevention Fellowship Program, Office of Preventive Oncology, $\mathrm{NCl}, \mathrm{NIH}$, Bethesda, MD, USA. ${ }^{3}$ Radiation Biology Branch, CCR, NCl, NIH, Bethesda, MD, USA. ${ }^{4}$ Gradient Corporation, Cambridge, Massachusetts, USA. ${ }^{5}$ Pathology and Laboratory Medicine, Baltimore Veterans Affairs Medical Center, Baltimore, MD, USA.
\end{abstract}

\section{Author's contributions}

SAG conceived and designed the study, performed experiments, participated in data collection, analyzed the data, and drafted the manuscript. RLP contributed to the study design, performed experiments, and analyzed data. LAR participated in study design and carried out experiments. BJB participated in study design, contributed to data collection, and analyzed data. TMD participated in data collection and carried out experiments. DAW participated in study design and manuscript preparation. JEG participated in data analysis and manuscript preparation. HGY performed the pathology and histology evaluation of the tissues and scored the

immunohistochemistry. DHL performed the pathology and histology evaluation of the tissues and evaluated immunohistochemistry. SA designed the study, contributed to data collection and analysis, and drafted the manuscript. All authors read and approved the final manuscript.

\section{Competing interests}

The authors declare that they have no competing interests.

Received: 1 March 2010 Accepted: 15 November 2010

Published: 15 November 2010

\section{References}

1. Howe LR, Chang SH, Tolle KC, Dillon R, Young LJ, Cardiff RD, et al: HER2/ neu-induced mammary tumorigenesis and angiogenesis are reduced in cyclooxygenase-2 knockout mice. Cancer Res 2005, 65:10113-10119.

2. Half E, Tang XM, Gwyn K, Sahin A, Wathen K, Sinicrope FA: Cyclooxygenase-2 expression in human breast cancers and adjacent ductal carcinoma in situ. Cancer Res 2002, 62:1676-1681.

3. Takeshita E, Osanai T, Higuchi T, Soumaoro LT, Sugihara K: Elevated cyclooxygenase-2 expression is associated with histological grade in invasive ductal breast carcinoma. J Med Dent Sci 2005, 52:189-193.

4. Costa C, Soares R, Reis-Filho JS, Leitao D, Amendoeira I, Schmitt FC: Cyclooxygenase 2 expression is associated with angiogenesis and lymph node metastasis in human breast cancer. J Clin Pathol 2002, 55:429-434.

5. Ristimaki A, Sivula A, Lundin J, Lundin M, Salminen T, Haglund C, et al: Prognostic significance of elevated cyclooxygenase-2 expression in breast cancer. Cancer Res 2002, 62:632-635.

6. Zerkowski MP, Camp RL, Burtness BA, Rimm DL, Chung GG: Quantitative analysis of breast cancer tissue microarrays shows high cox-2 expression is associated with poor outcome. Cancer Invest 2007, 25:19-26.

7. Park K, Han S, Shin E, Kim HJ, Kim JY: Cox-2 expression on tissue microarray of breast cancer. Eur J Surg Oncol 2006, 32:1093-1096.

8. Surowiak P, Materna V, Matkowski R, Szczuraszek K, Kornafel J, Wojnar A, et al: Relationship between the expression of cyclooxygenase 2 and MDR1/P-glycoprotein in invasive breast cancers and their prognostic significance. Breast Cancer Res 2005, 7:R862-R870.

9. Prueitt RL, Boersma BJ, Howe TM, Goodman JE, Thomas DD, Ying L, et al: Inflammation and IGF-I activate the Akt pathway in breast cancer. Int J Cancer 2007, 120:796-805.

10. Zhou X, Tan M, Stone HV, Klos KS, Lan KH, Yang Y, et al: Activation of the Akt/mammalian target of rapamycin/4E-BP1 pathway by ErbB2 overexpression predicts tumor progression in breast cancers. Clin Cancer Res 2004, 10:6779-6788.

11. Basu GD, Pathangey LB, Tinder TL, Lagioia M, Gendler SJ, Mukherjee P: Cyclooxygenase-2 inhibitor induces apoptosis in breast cancer cells in an in vivo model of spontaneous metastatic breast cancer. Mol Cancer Res 2004, 2:632-642.

12. Dirix LY, Ignacio J, Nag S, Bapsy P, Gomez H, Raghunadharao D, et al: Treatment of advanced hormone-sensitive breast cancer in postmenopausal women with exemestane alone or in combination with celecoxib. J Clin Oncol 2008, 26:1253-1259.

13. Canney PA, Machin MA, Curto J: A feasibility study of the efficacy and tolerability of the combination of Exemestane with the COX-2 inhibitor Celecoxib in post-menopausal patients with advanced breast cancer. Eur J Cancer 2006, 42:2751-2756.

14. Chow LW, Yip AY, Loo WT, Lam CK, Toi M: Celecoxib anti-aromatase neoadjuvant (CAAN) trial for locally advanced breast cancer. J Steroid Biochem Mol Biol 2008, 111:13-17.

15. Weidner N, Semple JP, Welch WR, Folkman J: Tumor angiogenesis and metastasis-correlation in invasive breast carcinoma. N Engl J Med 1991, 324:1-8.

16. Boersma BJ, Howe TM, Goodman JE, Yfantis HG, Lee DH, Chanock SJ, et al: Association of breast cancer outcome with status of p53 and MDM2 SNP309. J Natl Cancer Inst 2006, 98:911-919. 
17. Sorlie T, Perou CM, Tibshirani R, Aas T, Geisler S, Johnsen H, et al: Gene expression patterns of breast carcinomas distinguish tumor subclasses with clinical implications. Proc Natl Acad Sci USA 2001, 98:10869-10874.

18. Falandry C, Debled M, Bachelot T, Delozier T, Cretin J, Romestaing P, et al: Celecoxib and exemestane versus placebo and exemestane in postmenopausal metastatic breast cancer patients: a double-blind phase III GINECO study. Breast Cancer Res Treat 2009, 116:501-508.

19. Witton CJ, Hawe SJ, Cooke TG, Bartlett JM: Cyclooxygenase 2 (COX2) expression is associated with poor outcome in ER-negative, but not ERpositive, breast cancer. Histopathology 2004, 45:47-54.

20. Offersen BV, Alsner J, Ege OK, Riisbro R, Brunner N, Sorensen FB, et al: A comparison among HER2, TP53, PAI-1, angiogenesis, and proliferation activity as prognostic variables in tumours from 408 patients diagnosed with early breast cancer. Acta Oncol 2008, 47:618-632.

21. Ross JS, Fletcher JA, Linette GP, Stec J, Clark E, Ayers M, et al: The Her-2/ neu gene and protein in breast cancer 2003: biomarker and target of therapy. Oncologist 2003, 8:307-325.

22. Hicks DG, Short SM, Prescott NL, Tarr SM, Coleman KA, Yoder BJ, et al: Breast cancers with brain metastases are more likely to be estrogen receptor negative, express the basal cytokeratin CK5/6, and overexpress HER2 or EGFR. Am J Surg Pathol 2006, 30:1097-1104.

23. Heitz F, Harter P, Lueck HJ, Fissler-Eckhoff A, Lorenz-Salehi F, ScheilBertram S, et al: Triple-negative and HER2-overexpressing breast cancers exhibit an elevated risk and an earlier occurrence of cerebral metastases. Eur J Cancer 2009, 45:2792-2798.

24. Modi S, DiGiovanna MP, Lu Z, Moskowitz C, Panageas KS, Van Poznak C, et al: Phosphorylated/activated HER2 as a marker of clinical resistance to single agent taxane chemotherapy for metastatic breast cancer. Cancer Invest 2005, 23:483-487.

25. Emi Y, Kitamura K, Shikada Y, Kakeji Y, Takahashi I, Tsutsui S: Metastatic breast cancer with HER2/neu-positive cells tends to have a morbid prognosis. Surgery 2002, 131:S217-S221.

26. O'Donovan N, Crown J: EGFR and HER-2 antagonists in breast cancer. Anticancer Res 2007, 27:1285-1294.

27. Tokunaga E, Kimura Y, Oki E, Ueda N, Futatsugi M, Mashino K, et al: Akt is frequently activated in HER2/neu-positive breast cancers and associated with poor prognosis among hormone-treated patients. Int J Cancer 2006, 118:284-289.

28. Schmitz KJ, Otterbach F, Callies R, Levkau B, Holscher M, Hoffmann O, et al: Prognostic relevance of activated Akt kinase in node-negative breast cancer: a clinicopathological study of 99 cases. Mod Pathol 2004, 17:15-21.

29. Knuefermann C, Lu Y, Liu B, Jin W, Liang K, Wu L, et al: HER2/PI-3K/Akt activation leads to a multidrug resistance in human breast adenocarcinoma cells. Oncogene 2003, 22:3205-3212.

30. Kucab JE, Lee C, Chen CS, Zhu J, Gilks CB, Cheang M, et al: Celecoxib analogues disrupt Akt signaling, which is commonly activated in primary breast tumours. Breast Cancer Res 2005, 7:R796-R807.

31. Howe LR, Subbaramaiah K, Patel J, Masferrer JL, Deora A, Hudis C, et al: Celecoxib, a selective cyclooxygenase 2 inhibitor, protects against human epidermal growth factor receptor 2 (HER-2)/neu-induced breast cancer. Cancer Res 2002, 62:5405-5407.

32. Lanza-Jacoby S, Miller S, Flynn J, Gallatig K, Daskalakis C, Masferrer JL, et al: The cyclooxygenase- 2 inhibitor, celecoxib, prevents the development of mammary tumors in Her-2/neu mice. Cancer Epidemiol Biomarkers Prev 2003, 12:1486-1491.

33. Wang SC, Lien HC, Xia W, Chen IF, Lo HW, Wang Z, et al: Binding at and transactivation of the COX-2 promoter by nuclear tyrosine kinase receptor ErbB-2. Cancer Cell 2004, 6:251-261.

34. Subbaramaiah K, Norton L, Gerald W, Dannenberg AJ: Cyclooxygenase-2 is overexpressed in HER-2/neu-positive breast cancer: evidence for involvement of AP-1 and PEA3. J Biol Chem 2002, 277:18649-18657.

35. Benoit V, Relic B, Leval XX, Chariot A, Merville MP, Bours V: Regulation of HER-2 oncogene expression by cyclooxygenase-2 and prostaglandin E2. Oncogene 2004, 23:1631-1635.

36. Furuya F, Hanover JA, Cheng SY: Activation of phosphatidylinositol 3kinase signaling by a mutant thyroid hormone beta receptor. Proc Natl Acad Sci USA 2006, 103:1780-1785.

37. Andjelkovic M, Alessi DR, Meier R, Fernandez A, Lamb NJ, Frech M, Cron P, Cohen P, Lucocq JM, Hemmings BA: Role of translocation in the activation and function of protein kinase B. J Biol Chem 1997, 272:31515-31524.

38. Scheid MP, Marignani PA, Woodgett JR: Multiple phosphoinositide 3kinase-dependent steps in activation of protein kinase B. Mol Cell Biol 2002, 22:6247-6260.

\section{Pre-publication history}

The pre-publication history for this paper can be accessed here: http://www.biomedcentral.com/1471-2407/10/626/prepub

doi:10.1186/1471-2407-10-626

Cite this article as: Glynn et al:: COX-2 activation is associated with Akt phosphorylation and poor survival in ER-negative, HER2-positive breast cancer. BMC Cancer 2010 10:626.

\section{Submit your next manuscript to BioMed Central and take full advantage of:}

- Convenient online submission

- Thorough peer review

- No space constraints or color figure charges

- Immediate publication on acceptance

- Inclusion in PubMed, CAS, Scopus and Google Scholar

- Research which is freely available for redistribution

Submit your manuscript at www.biomedcentral.com/submit
Biomed Central 